\title{
CONTROL OF THE FLOW STRUCTURE OF A CIRCULAR CYLINDER USING CONCENTRICALLY INSTALLED PERFORATED CYLINDER AND SPLITTER PLATE
}

\author{
${ }^{* 1}$ Engin Pınar ${ }^{2}$ Arda Yeniçun ${ }^{3}$ Göktürk M.Özkan ${ }^{4}$ Tahir Durhasan ${ }^{3}$ Umutcan OLMUŞ ${ }^{3}$ Hüseyin Akılll ${ }^{3}$ Beşir \\ Şahin \\ ${ }^{1}$ Faculty of Ceyhan Engineering, Department of Mechanical Engineering Çukurova University, Turkey \\ ${ }^{2}$ Karaisalı Vocational School, Çukurova University, Turkey \\ ${ }^{3}$ Faculty of Engineering, Department of Mechanical Engineering Çukurova University, Turkey \\ ${ }^{4}$ Faculty of Aeronautics and Astronautics, Department of Aerospace Engineering, Adana Science and \\ Technology University, Turkey
}

\begin{abstract}
The purpose of this work was to control undesired structure and vibrations caused from vortex shedding by controlling the flow downstream of the cylinder. The porosities of the outer cylinder and the splitter plate used for this purpose are available. The other parameter was plate angle based on the flow direction as a reference. The parameter expressed as porosities, $\beta$ was chosen to have a $\% 70$ openness for the outer cylinder $(\beta=$ 0.7 ) and was kept constant throughout all experiments. On the other hand, two different porosities were used for the splitter plate. For the first plate, there was no hole on the plate which we call $\beta=0$ or solid plate. For the second plate, it has $\beta=0.7$ porosity. $\beta=0.7$ porosity was also the value chosen for the outer cylinder. The range of the splitter plate angle was selected within $60^{\circ} \leq \alpha \leq 180^{\circ}$ with an increment of $30^{\circ}$. The results were extracted using two different methods. The first was the dye visualization experiment and the second is the PIV (Particle Image Velocimetry) measurement. As a result, it was observed that independently from the outer cylinder effect, the plate porosity and plate angle are effective on the level of turbulence and the flow structure in the annular region.
\end{abstract}

Key Words: Circular cylinder, TKE, Passive flow control, Splitter plate

\section{Introduction}

The flow structures that form around the circular cylinder resulting flow separations on shear layer and vortex shedding and vibration cause by these shedding have attracted many researchers for years. The vortex shedding behaviors in downstream of the circular cylinder have found itself a place in many articles because of their use in real engineering applications and their easy handling. Mechanical and energy application such as turbine blades, heat exchanger tubes, civil engineering applications such as chimneys, bridges and offshore structure, underwater applications such as underwater pipelines, marine cables can be an example of these areas that uses principles of flow over circular cylinder. The reason for the studies draws such interest on itself is that both the aerodynamic and the hydrodynamic field data are useful in aforementioned applications. The factors that can influence flow separation downstream of the cylinder are not only because of circular cylinders but also the number of Reynolds numbers. The experimental studies and applications of real-life engineering show that vortex formation due to flow separation causes abundance effect on the cylinder such as structural vibrations caused vortex shedding, noise due to this vibration and even decrease structure life of body. The simplest and easiest method is the passive flow control method used 
to prevent the above-mentioned effects. The passive flow control methods are not time dependent and do not require external energy input. If passive methods do not need energy from outside, how does it suppress or decrease vortex shedding? The answer to this question is hidden in changing the geometry of the body and adding external devices along the body. In this study, one the passive flow control methods, the perforated outer cylinder and perforated splitter plate were used together. Although the water tunnel was used in this study, in general, the vortex formation over a circular cylinder was examined in two different tunnels which are air and water tunnel. Many academic studies have been carried out to examine the flow patterns and flow behaviors downstream of the cylinder. The use of circular cylinder is due to the fact that cylinder is the basic forms used in real engineering applications. In the previous studies, although the bodies made of spherical cone and square sections body were examined, they attracted less attention than the circular cylinders. In the light of the importance of circular cylinder, firstly, those who draw attention from early studies should be examined $[1,2]$. These studies showed that the circular cylinder shape body could encountered the interactions between the two shear layers separated from either side of the cylinder, and hence lead to the vortex shedding in the downstream of the cylinder. There are some studies in the literature to control the non-perturbed flow structure formed downstream of the cylinder using perforated or porous structures. In his dissertation on geometric shape changes in flow control, [3] he used various shapes of outer shirts (shrouds) to control vortex-induced vibrations. Because these shirts delayed the formation of the Karman vortices, they noted that an object exposed to the flow reduced vortex shedding of an object. Every, King [4] in the compilation he made, he studied the work done up to that time on the control of vortex-induced vibrations, especially in submarine applications. In one part of the review, it was noted that an outer shirt with optimum dimensions placed on the direction of flow, reducing the oscillations of the body by $50 \%$. It is not only experimental but also numerical studies where a perforated outer structure is used. Bruneau and Mortazavi [5] made a good research that could be a reference to this study because of the point of view of idea of using perforated outer structure. They numerically examine the effects of porous sheathing around a pipe section. They performed their observations at $\operatorname{Re}=$ 15000 and $\mathrm{Re}=15 \times 10^{4}$, which are close to the actual sea conditions. They noted that the presence of the porous layer reduces the shear effects on the boundary layer and thus changes the vortex break positively. In addition, they have found that this passive control reduces $\mathrm{C}_{\text {Lrms }}$ to $55 \%$ for the Reynolds number, $\mathrm{Re}=15 \times 10^{3}$ and to $72 \%$ for the Reynolds number, $\mathrm{Re}=$ $15 \times 10^{4}$. The Particle Image Velocimetry (PIV) technique is important because this technique can be adaptable in underwater applications. In this study most of the results were found using the PIV technique. In this context In this context, it would be wise to look at the work that used this technique before. Prasad and Williamson [6] is leading investigation on a study that used Particle Image Velocimetry (PIV) technique with the cylindrical body. Ozkan, Oruc [7] was aimed to control the flow around a circular cylinder with the perforated cylinder having four different porosity ratios $(\beta=0.4,0.5,0.6,0.7)$ and diameter ratios $(\mathrm{D} / \mathrm{d}=1.2,1.4,1.6,1.8,2.0)$. They used a cylinder with a diameter of $100 \mathrm{~mm}$ and a permeability ratio of $\beta=0.7$ for flow control in a $50 \mathrm{~mm}$ diameter circular cylinder. They concluded that the effects on the flow behavior of the cylinders placed concentrically around the inner cylinder to be controlled were investigated experimentally in shallow water by means of particle image velocity measurement (PIV) and were significantly effective on the flow characteristics of the permeable outer cylinder. According to the experimental results, the permeable outer cylinder reduced the vortex values by suppressing the vortex structure formed by the inner cylinder and also significantly reduced the turbulence statistics (Reynolds stresses and turbulence kinetic energy). Bengi Gözmen [8] aim to investigate the effect of the porosity ratio of outer cylinder to the flow of downstream of a circular inner cylinder, working with eight different porosity ratios $(\beta=0,4$, $0,5,0,6,0,65,0,7,0,75,0,8$ and 0,85$)$. Among the all eight different porosity ratios, they 
concluded that perforated outer cylinder, have $\beta=0,7$ ratio is the most useful parameter to control the flow downstream of the circular cylinder. At this porosity ratio suppressed Von Karman Vortex Street and also the momentum transfer from the free stream flow into the wake region is prevented. Akilli, Sahin [9] experimentally studied the effect of splitter plates which are not connected to the cylinder, with varying positions on the suppression of vortex shedding in shallow water with PIV techniques. Although the length of splitter plates remains constant at $50 \mathrm{~mm}$, plates have different thickness. They conducted experiments with $50 \mathrm{~mm}$ diameter of cylinder $(D)$ which is equal to the length of splitter plates. Gap distance between the base of the cylinder and the leading edge of the splitter plate, time-averaged velocity vector fields for various cases of gap ratio $(G / D)$ and plate thickness ratio $(T / D)$ were used to understand the effect of plate thickness on the suppression of vortex shedding. Although, the splitter plate has a substantial effect on suppression of the vortex shedding for the gap ratio between 0 and $1.75 \mathrm{D}$, no effect of the splitter plate was observed when the splitter plate was located at 2D location. They showed that altering the flow characteristic plate thickness had same effect. After passing the splitter plate, the flow starts to oscillate with smaller frequency compared to the natural frequency of the bare cylinder. Ozkan, Firat [10] have examined the effect of splitter plates with various porosity ratios on the drift coefficient. The parameter they intended to investigate was the effect of the porosity ratio of the splitter plate $(\beta)$ mounted at a certain angle according to the flow. According to this definition values of $\beta$ founded as $\beta=0.4,0.5,0.6,0.7$ and 0.8 . Except $\beta=0.7,0.8$ case, when the other porosity ratios of plate are used drag coefficient ascend. Besides this, the situation is slightly different for $\beta=0.7,0.8$ in cases where plates with this permeability ratio are used, the success in preventing vortex shedding has not been achieved, but a good contribution has been made in terms of vibration and fluctuation acting on cylinder.

The result from all these studies combines that $\beta=0.7$ porosity ratio is a critical value. Therefore, $\beta=0.7$ porosity ratio both outer cylinder and splitter plate was chosen for this study although there were differences in experimental setup and experimental parameters. Furthermore, while the experiments and studies conducted are mostly focused on the effects area downstream of the circular inner cylinder, in this study the effect on the area between the inner and outer cylinder (annular region) will be the main purpose.

\section{Materials and Method}

This work was carried out in closed loop open water channel as seen in Figure 1 established at Çukurova University, Mechanical Engineering Department, Fluid Mechanics Laboratory. The water channel consisted two water reservoirs with an area of $750 \mathrm{~mm} \mathrm{x}$ $1000 \mathrm{~mm} \times 8000 \mathrm{~mm}$ (height $\mathrm{x}$ width $\mathrm{x}$ height) located between these two reservoirs. There was a 2: 1 contraction section to ensure the passage between the inlet water reservoir and the experimental section. Both the PIV measurement and dye visualization experiments were performed in this water channel. The velocity of water changed when necessary by a $15 \mathrm{~kW}$ centrifugal pump which has an electrical driver to adjust frequency.

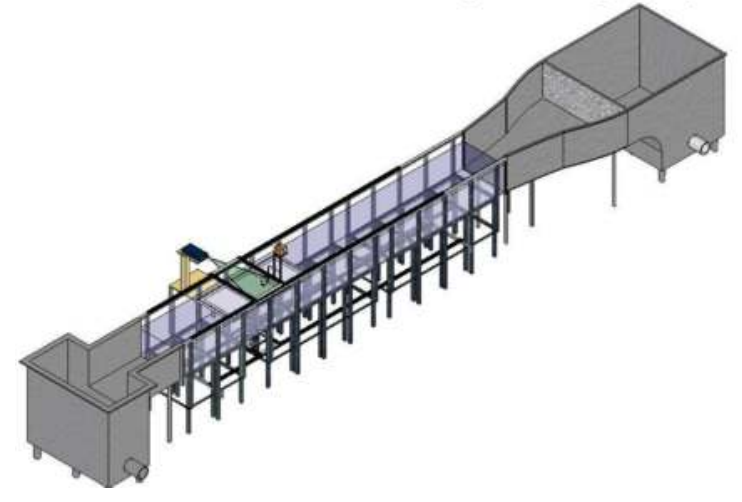


Figure 1 Water Channel

The free stream velocity was taken as $100 \mathrm{~mm} / \mathrm{s}$ which corresponds to the Reynolds number of $\mathrm{Re}_{\mathrm{d}}=5000$ according to the cylinder. During all experiments, the total depth of the water in the channel was kept constant as $600 \mathrm{~mm}$. As schematic view of the experimental setup is shown in Figure 2.

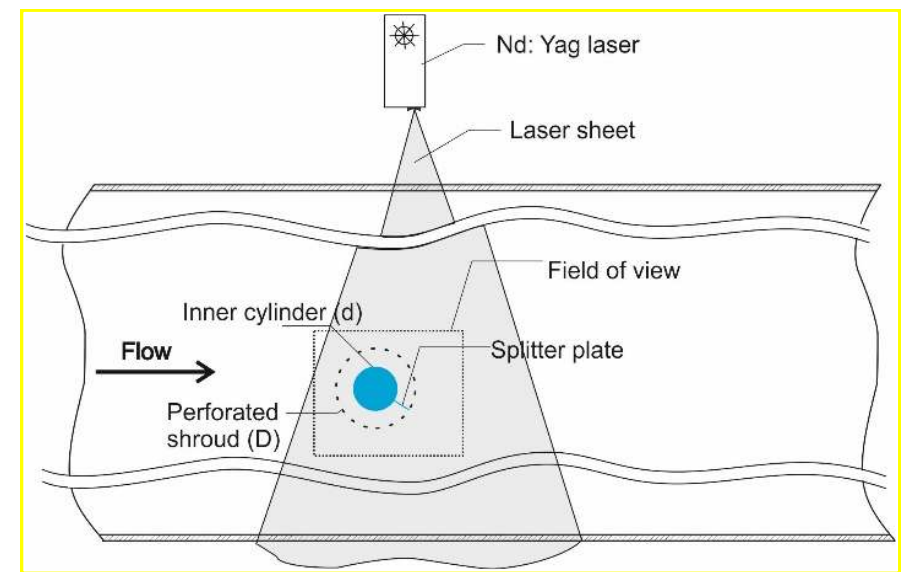

Figure 2 Top view of the experimental set-up

The geometric blockage of the perforated cylinder was $10 \%$ with respect to the width of test section. Chrome-nickel metals sheets with $1 \mathrm{~mm}$ thicknesses were drilled using laser cutting machine to have $d=10 \mathrm{~mm}$ holes on the cylinder surface, then they were rolled to manufacture perforated shroud. The porosities, $\beta$ which was defined as the ratio of the total open area on the cylinder to the whole cylinder surface area was chosen as a dimensionless parameter because the number of the holes on the shroud had significant effects on the flow structure. Figure 3 shows the schematic representation of the dimensions of solid plate, inner cylinder diameter $(d=50 \mathrm{~mm})$ and the perforated shroud $(\mathrm{D}=125 \mathrm{~mm})$. The porosity, $\beta$ value was taken constant as $\beta=0.7$ through the experiments.

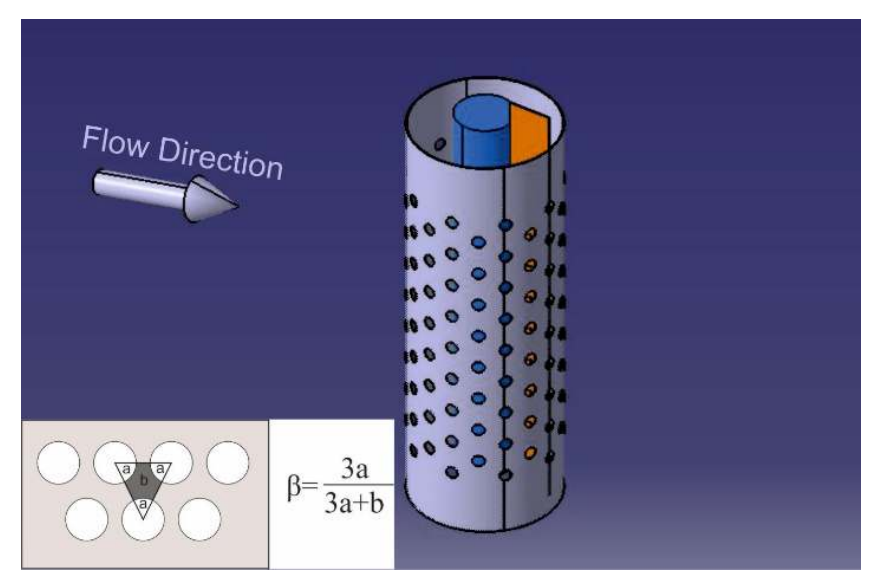

Figure 3 Schematic representation for the control method and the definition for the porosity, $\beta$

The laser sheet was adjusted parallel to the upper surface of the platform at a height of $25 \mathrm{~mm}$ which is half of the water level. Experiments were conducted in two steps: In the first step, the flow visualization experiments were performed using Rhodamine type dye that shines under the continuous laser beam in the desired flow field. Throughout the experiments, dye was 
supplied at a location where the flow was not affected by the injection. Visualization of the experiment al results was recorded with a high-speed SONY 80X handycam type digital video recorder to examine the flow behaviors in detail. (Figure 4)

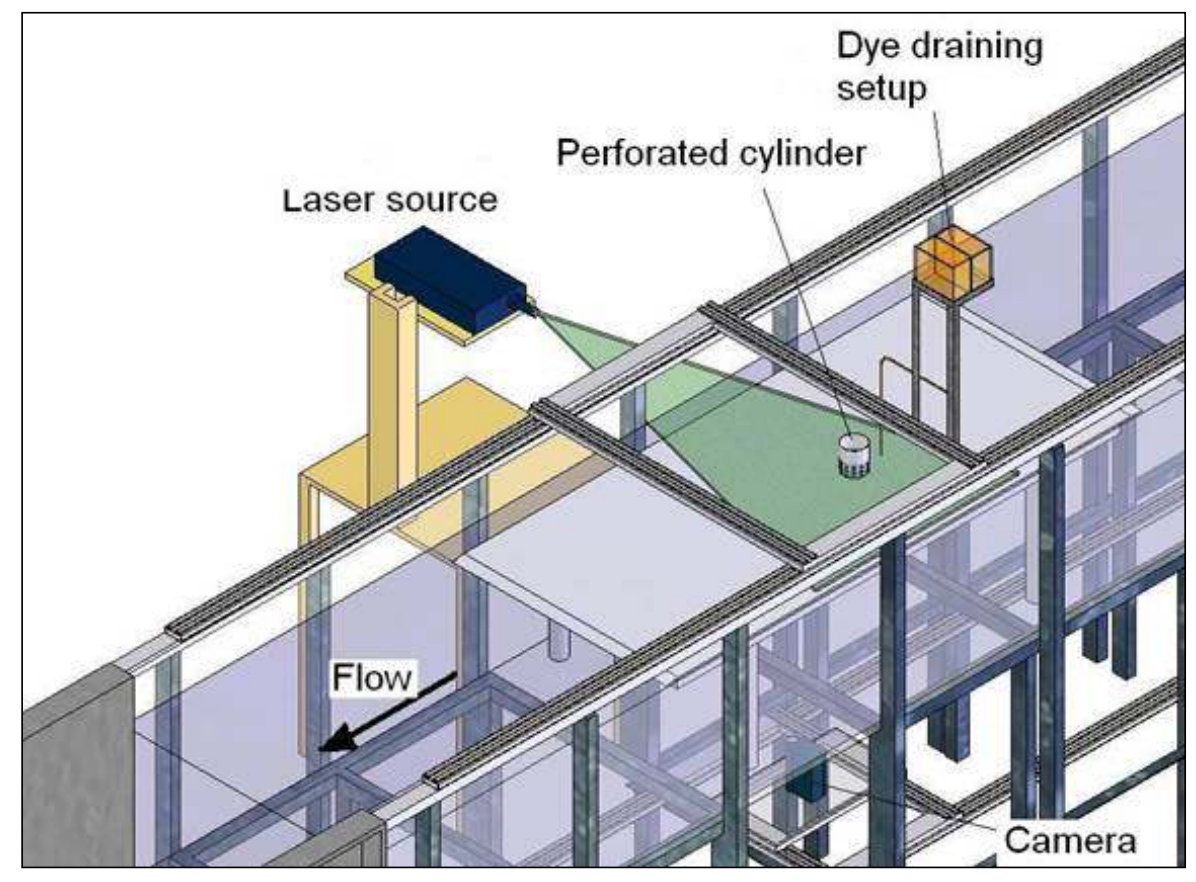

Figure 4 Schematic representation of dye experimental method.

In the second step, the Particle Image Velocimetry (PIV) technique was used in order to measure the velocity vector fields. Experiments were focused on area between the cylinder and perforated shroud (which will be called the annular region) for all porosity by PIV technique in order to see the effect of porosity on the flow structure in gap area section of the cylinders since the dye visualization experiments were not enough to understand flow structure completely because of accumulation of dye in restricted area. The measurement field was illuminated by a thin and intense laser light sheet by using a pair of double-pulsed Nd:YAG laser units. Each pulse has a nominal energy output of $120 \mathrm{~mJ}$ at $532 \mathrm{~nm}$ wave length. The time interval between pulses was $1.5 \mathrm{~ms}$ for all experiments and the thickness of the laser sheet illuminating the measurement plane was approximately $2 \mathrm{~mm}$. The time interval and the laser sheet thickness were selected such that the maximum amount of particle displacement in the interrogation window was obtained. The water was seeded with $10 \mu \mathrm{m}$-diameter seeding particles and the particle density was about $1100 \mathrm{~kg} / \mathrm{m}^{3}$. The number of particles in an interrogation area was in between 15 and 20. The experimental measurements were performed and the data were processed using Dantec Dynamics PIV system and Dynamics Studio Software installed on a computer. In each experiment, 1000 instantaneous images were captured at the rate of $15 \mathrm{~Hz}$, recorded and stored in order to obtain time-averaged velocity vectors, vorticity contours, kinetic energy contours. Assuming isotropic flow, the values of turbulent kinetic energy (TKE) were evaluated using the estimation of Sheng, Meng [11] which states that the third component has been supposed to be equal to the half of $\left(\left\langle u^{\prime} u^{\prime}\right\rangle+\left\langle v^{\prime} v^{\prime}\right\rangle\right)$ and TKE can be calculated using $\mathrm{TKE}=3 / 4\left(\left\langle u^{\prime} u^{\prime}\right\rangle+\left\langle v^{\prime} v^{\prime}\right\rangle\right)$. The image capturing was performed by an 8-bit cross-correlation CCD camera having a resolution of $1600 \times 1200$ pixels, equipped with a Nikon AF Micro 60 $\mathrm{f} / 2.8 \mathrm{D}$ lens. In the image processing, $32 \times 32$ rectangular interrogation pixels were used and an overlap of $50 \%$ was employed. Shadows, reflections or laser sheet distortions etc. cause spurious velocity vectors in the flow field, local median-filter technique was employed to erase 
spurious vectors (less than 3\%) in which they were replaced using bilinear interpolation between neighbouring velocity vectors. The velocity vector field was also smoothed to avoid dramatic changes in the velocity field using the Gaussian smoothing technique. To minimize distortion of the velocity field, a smoothing parameter of 1.3 was chosen. The details of the effects of these factors can be found in the studies of Fouras and Soria [12], Adrian [13] and Keane and Adrian [14]. Finally, the instantaneous and mean vorticity maps (vorticity value at each grid point was calculated from the circulation around eight neighbouring point) and turbulence statistics were determined as a result of post-processing operation.

\section{Results and Discussions}

\subsection{Dye Visualization Technique}

Flow visualization experiments have been carried out in detail in this interval and the results have been examined. First of all, it should be noted that the visualization of the experiments result and flow structure can be observed while watching videos, but it is difficult to observe the flow structures when images are taken from these videos. The direction and behavior are shown with red arrows by following the videos obtained by this reason. Figure 5 shows the results of these dye visualization experiments and red lines show that flow structures and behaviors. Only the outer cylinder and inner-outer cylinder pairs are given in the top row of Figure 5. It has been observed that the discharge from the openings and holes is formed nothing but turbulent flow because the flow rate through the holes is high. In the case of the inner-outer cylinder couple, a couple of vortices were formed in the region downstream of the inner cylinder. It is presumed that these vortices that form downstream of the inner cylinder will inevitable damage on the inner cylinder and should be controlled for this reason. In Figure 5 , the solid plate which can be also named $\beta=0$ splitter plate, for $\alpha=180^{\circ}$, it is observed that the resulting vortex pairs are separated from each other. Between the $60^{\circ} \leq \alpha \leq 120^{\circ}$ plate angles, solid plate blocked the flow in the annular region. Due to this blockage effect of the flat plate, reasonable physical flow structures, which are also shown by red arrows at the edges of the other plate angles, have been obtained. It was seen in this angles that some amount of flow was directed outside the plate in other words lower shear layer of the cylinder while major amount of flow and momentum directed to the upper shear layer of inner cylinder. This situation is observed as the flow re-entered the annular region because of higher density of flow and higher momentum of upper shear layer. This can be said to be effective in reducing the turbulence intensity of the flow in the annular region, but quantitative results from the PIV will show this. When the solid plate angle comes to $\alpha=150^{\circ}$, the flow that directed to the outside of annular region and doesn't form vortex in the lower shear layer, at this angle forms a vortex in front of the solid plate. The angle value of $\alpha=180^{\circ}$ presents the well-known splitter plate method within the relevant literature and might be the best way of preventing the interaction of vortices formed by the inner cylinder within the annular region. Here the vortices are more distinct compared with the no-plate case and their interaction is eliminated.

The use of the splitter plate which has a $\beta=0.7$ porosity instead of a solid plate will allow one portion of flow to pass to the other side of the plate, rather than block the flow in annular region. In second column of Figure 5 , the plates with a porosity ratio of $\beta=0.7$ are affected by the flow in the annular region. Looking at the picture, it is understood that for almost all plate angles, the same flow structure occurs. It is evident from the flow visuals that for $\beta=0.7$, there is a vortex pair structure and turbulent flow structure in the annular region starting from angle $\alpha=60^{\circ}$. 


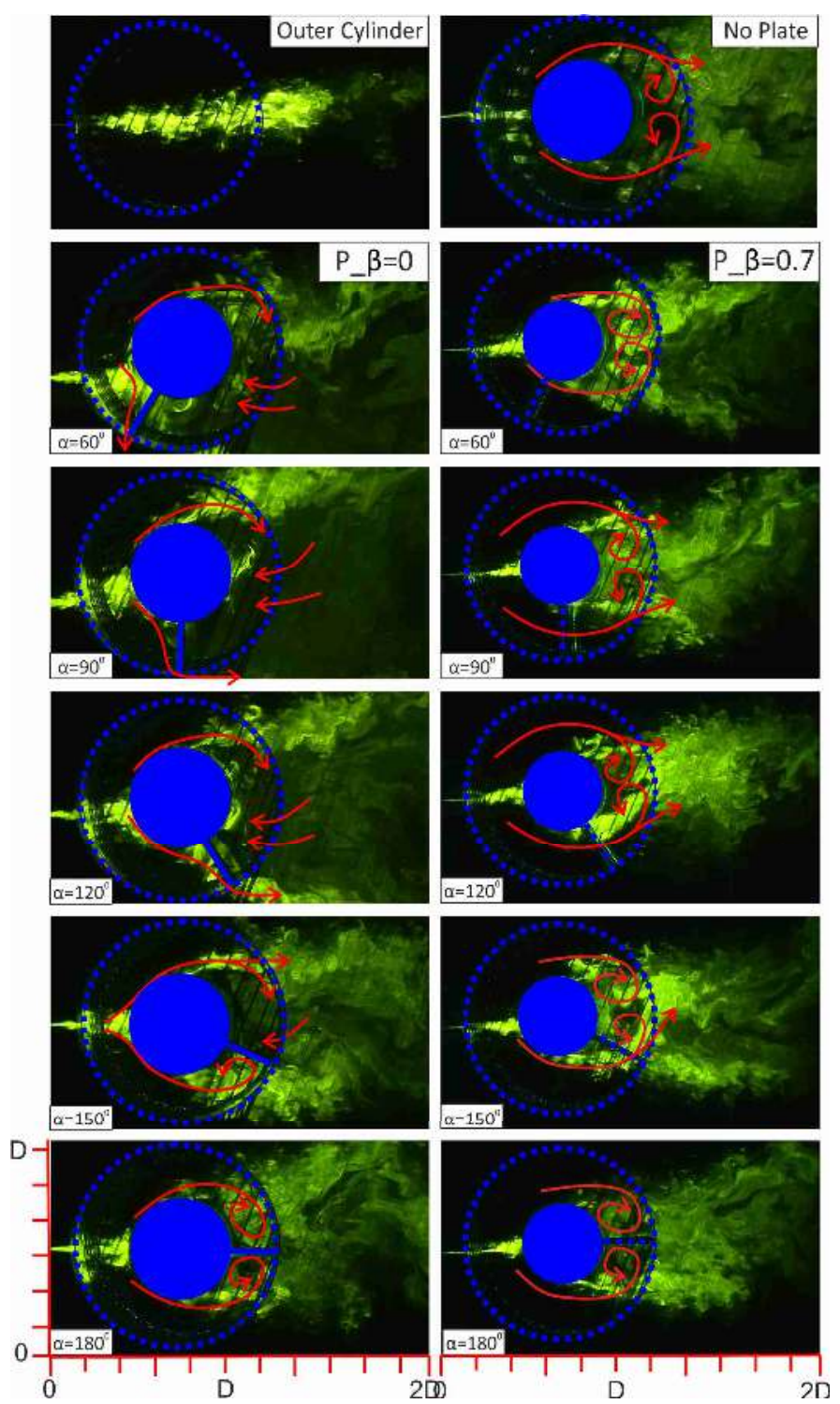

Figure 5 Dye visualization images of flow structure in annular region for porosity ratio $\beta=0.7$ outer cylinder and $\beta=0$ and $\beta=0.7$ splitter plate at different angle.

\subsection{PIV Technique}

PIV experiments were conducted to support the results of the observed flow structure after the dye visualization experiments and to quantitatively determine which parameters are more appropriate.

In Figure 7 and Figure 8, the time average streamlines $\langle\psi\rangle$ with the vorticity contours obtained for the case of only the $\beta=0.7$ outer cylinder, inner-outer cylinder couple and $\beta=0$ and $\beta=0.7$ splitter plate are shown, $<\omega>$. Figure 7 also shows the position of the free stop point and vortex focal points (F1, F2, F3 and F4) of the flow occurring downstream of the inner cylinder. While the circulation of the streamlines indicated by $\mathrm{F}$ is centered clockwise from the top half of the cylinder, the streamlines formed at the bottom half circulate in the opposite direction. In order to compare the influence of the control parameters on the flow structure downstream of the inner cylinder, in the case of only the perforated outer cylinder in the flow (no inner cylinder with diameter $\mathrm{Di}=50 \mathrm{~mm}$ ) and only the perforated outer cylinder as the control element. The time-averaged streamlines for $\mathrm{Di} / \mathrm{Dd}=0.4$ and the time-averaged vorticity contours $\langle\omega\rangle$ are shown. From the streamlines, $\langle\psi\rangle$ indicates that no focal points occur when the inner cylinder is absent. In the case of the inner-outer cylinder pair in the first 
row of second column, it is seen that the circulation shown by F1 and F2 is formed in the region between cylinder pair for $\beta=0.7$ outer cylinder. Plate porosity ratio $\beta=0$ and plate angle for between $\alpha=60^{\circ}$ and $\alpha=120$, the use of solid plate without hole as control element was effective on the flow structure. The results for these parameters are compared with the result obtained in the case where the plate is not used as the control element (first row and second column) in Figure 7. It is obvious that instead of the two F1 and F2 focal points occurring downstream of the inner cylinder, a single focal point with a smaller scale, $F_{1}$ occurs. When splitter plate porosity increases to $\beta=0.7$ two $F_{1}$ and $F_{2}$ focal points are occurs for all plate angle. Results are almost same both time average streamline and vorticity contour, in the case of plate porosity $\beta=0.7$. The most effective parameters for preventing the focal points formed downstream of the inner cylinder were determined as the plate porosity ratio is $\beta=0$ (solid plate) with angle between $\alpha=60^{\circ}$ and $\alpha=120$.

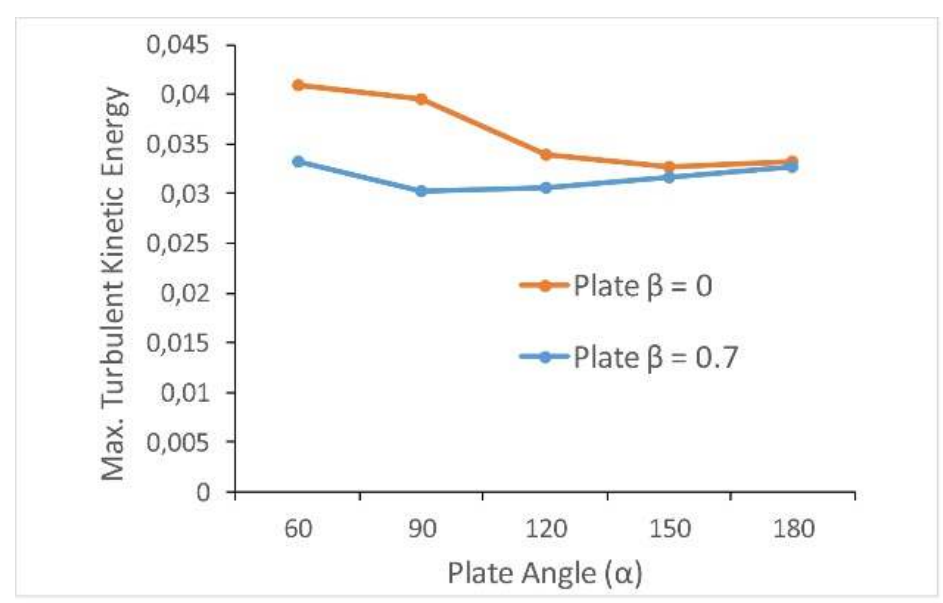

Figure 6 Change of the maximum TKE value for the plate porosity ratio $\beta=0$ and $\beta=0.7$ with plate angle, $\alpha$.

When the dye visualization experiments were examined, it was observed that vortices and circulation occurred in the annular region where between the inner and outer cylinder. These circulations are the reason why focal points are observed in the streamlines for many situations. Looking at the focal points, it is not possible to say that the control parameters are completely effective or ineffective. For these reasons, even though focal points occur in the annular region of the cylinder pair, there may be a decrease in turbulence statistical value such as Turbulence Kinetic Energy of the flow downstream of the inner cylinder. Turbulence Kinetic Energy (TKE) is calculated in our work because it gives more general information about the turbulence level that the flow has. Figure 6 shows change of the maximum TKE value with plate angle, $\alpha$. As the control member the maximum TKE value in the annular region was found at 0.031 when the case an outer cylinder with a porosity ratio $\beta=0.7$ was used. When the outer cylinder and the splitter plate are used together at $\alpha=60^{\circ}$ and $90^{\circ}$, the maximum TKE value is greater than the maximum TKE obtained when the plate angle is only the outer cylinder as the control element. It has been determined that the use of perforated splitter plate does not significant affect the maximum TKE values when only with the use of perforated cylinders, although the maximum TKE values decrease with increasing plate angle. In summary, for the porosity ratio $\beta=0.7$, it was determined that the perforated cylinder and plate were not an effective instead of using only a perforated cylinder as a control element from the perspective of TKE. 


\section{E. PINAR et al./ISITES2018 Alanya - Antalya - Turkey}

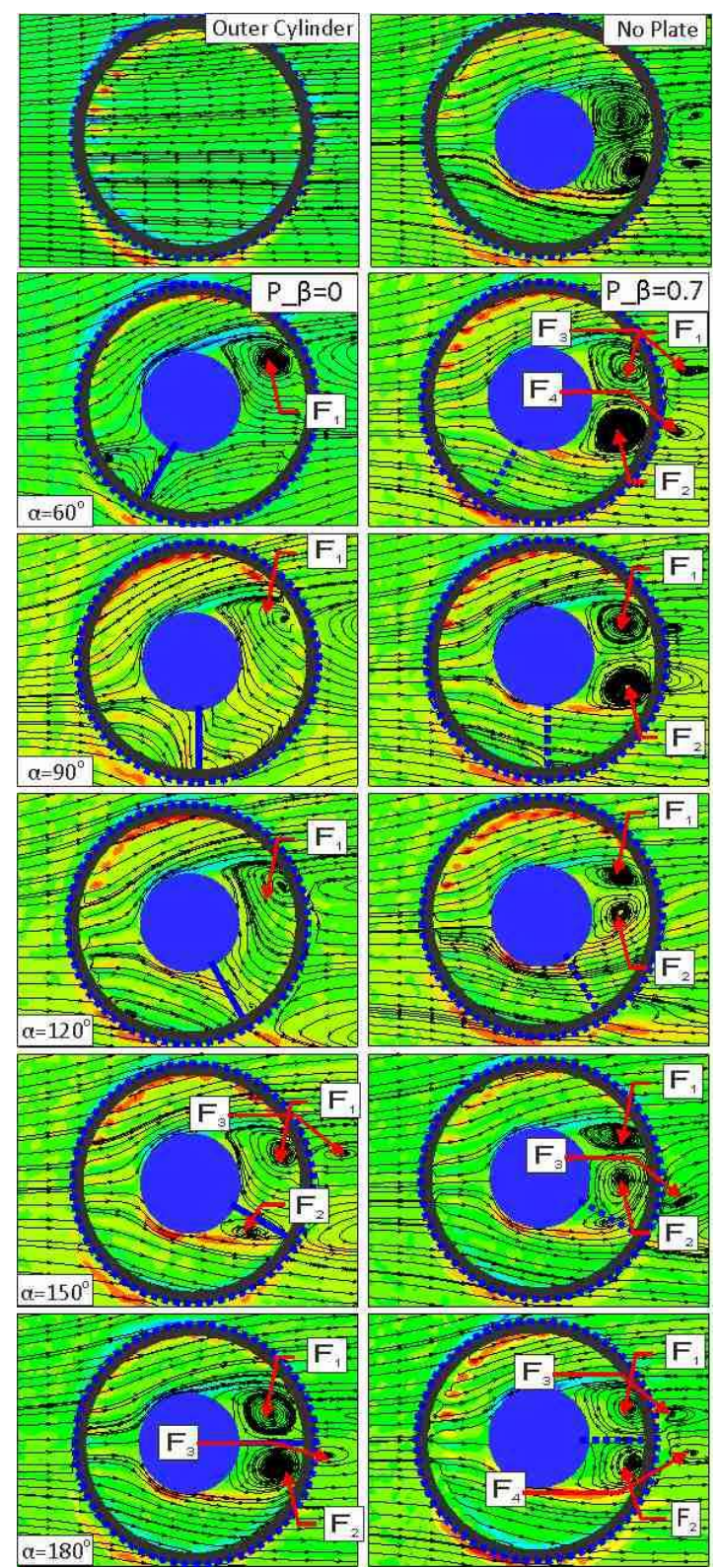

Figure 7 The time-averaged streamline, $<\Psi>$ with the vorticity contours all experiment parameters 


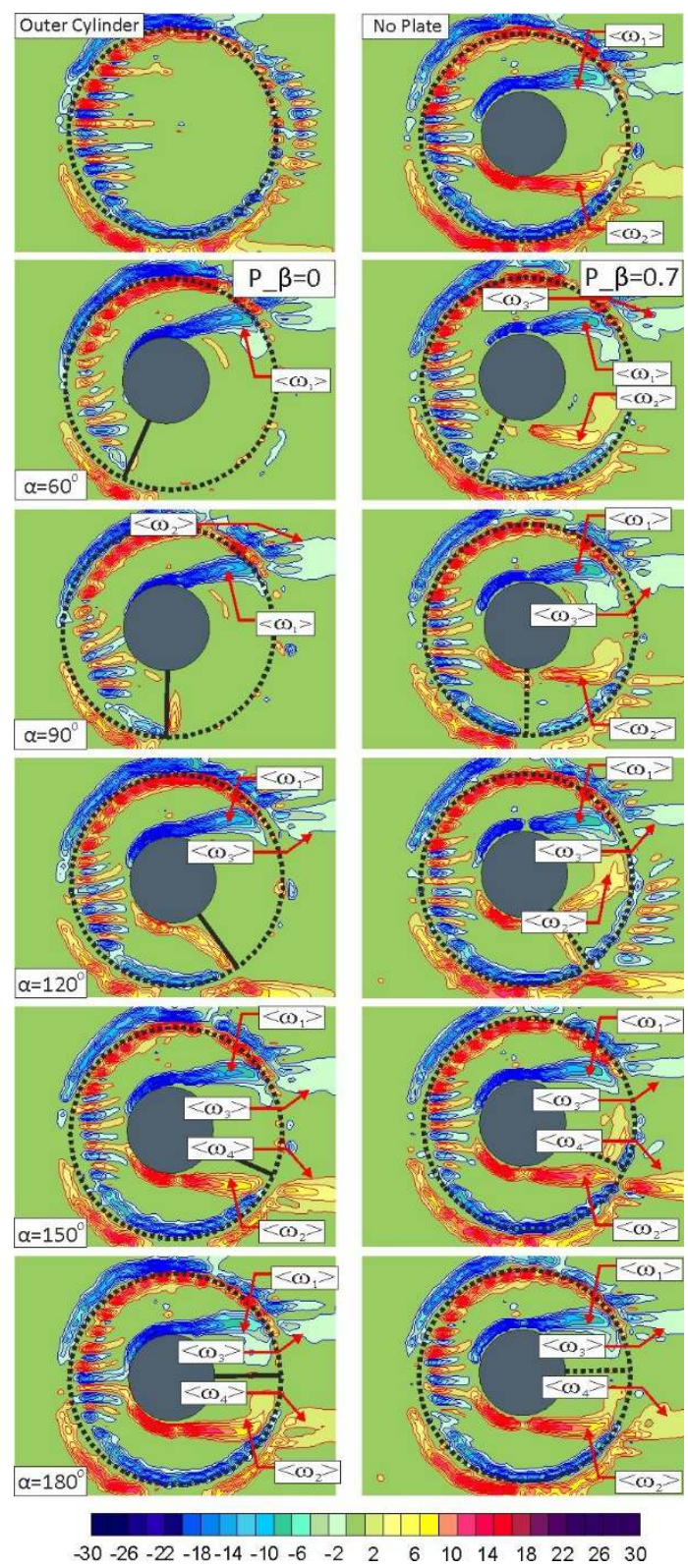

Figure 8 The time average vorticity contour $<\omega>$ for in the case of the outer cylinder porosity ratio $\beta=0.7$ with the different plate angles $\alpha$,

\section{Conclusions}

As a result of experiments with the particle imaging measurement technique, the flow structure was tried to be interpreted with the aim of determining the optimum parameters cultivated in the control of the continuous flow structure formed in the annular region by using time average streamlines $\langle\psi\rangle$ and time average vorticity contour $\langle\omega\rangle$. When the obtained images were examined, for many cases where the control elements (splitter plate at different angles) were used, results similar to the flow structure were obtained in the case where only the inner-outer cylinder couple is present in the flow. For example, in the case where only the cylinder couple was present in the flow, the focal points $F_{1}$ and $F_{2}$ formed in the separated flow region of the inner cylinder in many cases.

According to the results obtained from the experiments, it was determined that the use of $\beta=0$ plate is more effective in the reduction of the vortices in the annular region than in $\beta=$ 
0.7. However, it was seen from the graph that the TKE values are higher for $\beta=0$ under the same conditions. For the plate porosity $\beta=0$ and the plate angle range between $60^{\circ} \leq \alpha \leq 120^{\circ}$ condition, the vortex couple formed just downstream of the inner cylinder was converted into a single vortex which will cause undesirable vibrations on the inner cylinder. The best reduction in the turbulence was evaluated for the plate angle of $\alpha=90^{\circ}$ in which the turbulence concentrations were lower in the near wake than the case only the perforated outer cylinder. Therefore, the splitter plate angle of $\alpha=90^{\circ}$ could be advised for the current method. On the other hand, the plate angles of $\alpha=150^{\circ}$ and $\alpha=180^{\circ}$ could be suggested because they almost completely separate the vortex couple originating from the upper shear layer and the lower shear layer of the inner cylinder. However, despite these separated vortex couple, the high TKE values remain a predictive and worth research parameter. Therefore, it would be logical conduct future research in order to fully control the vortex shedding downstream of the cylinder with different parameter such as changing outer cylinder porosities or change the splitter plate width.

\section{References}

[1]. Roshko, A., On the drag and shedding frequency of two-dimensional bluff bodies. Tech. 1954, Note 3169. NACA, Washington.

[2]. Roshko, A., Experiments on the flow past a circular cylinder at very high Reynolds number. Journal of Fluid Mechanics, 1961. 10(3): p. 345-356.

[3]. Price, P., Suppression of the fluid-induced vibration of circular cylinders. Journal of the Engineering Mechanics Division, 1956. 82(3): p. 1-22.

[4]. Every, M., R. King, and D. Weaver, Vortex-excited vibrations of cylinders and cables and their suppression. Ocean Engineering, 1982. 9(2): p. 135-157.

[5]. Bruneau, C.-H. and I. Mortazavi, Control of vortex shedding around a pipe section using a porous sheath. International Journal of Offshore and Polar Engineering, 2006. 16(02).

[6]. Prasad, A. and C.H. Williamson, The instability of the separated shear layer from a bluff body. Physics of Fluids, 1996. 8(6): p. 1347-1349.

[7]. Ozkan, G.M., et al., Flow around a cylinder surrounded by a permeable cylinder in shallow water. Experiments in Fluids, 2012. 53(6): p. 1751-1763.

[8]. Bengi Gözmen , H.A., Beşir Şahin, Vortex control of cylinder wake by permeable cylinder. 2013.

[9]. Akilli, H., B. Sahin, and N. Filiz Tumen, Suppression of vortex shedding of circular cylinder in shallow water by a splitter plate. Flow Measurement and Instrumentation, 2005. 16(4): p. 211-219.

[10]. Ozkan, G.M., E. Firat, and H. Akilli, Passive flow control in the near wake of a circular cylinder using attached permeable and inclined short plates. Ocean Engineering, 2017. 134: p. $35-49$.

[11]. Sheng, J., H. Meng, and R. Fox, A large eddy PIV method for turbulence dissipation rate estimation. Chemical engineering science, 2000. 55(20): p. 4423-4434.

[12]. Fouras, A. and J. Soria, Accuracy of out-of-plane vorticity measurements derived from in-plane velocity field data. Experiments in fluids, 1998. 25(5-6): p. 409-430.

[13]. Adrian, R.J., Particle-imaging techniques for experimental fluid mechanics. Annual review of fluid mechanics, 1991. 23(1): p. 261-304.

[14]. Keane, R.D. and R.J. Adrian. Optimization of particle image velocimeters. in ICALEO'89: Optical Methods in Flow and Particle Diagnostics. 1990. International Society for Optics and Photonics. 